Editora Omnis Scientia

ANAIS DA I JORNADA NORTE-NORDESTE DE ENGENHARIAS (ONLINE)

Volume 1

$1^{\text {a }}$ Edição

TRIUNFO - PE 


\section{Coordenador Científico}

Alex Aguiar da Silva

\section{Coordenador de Publicação}

Daniel Luís Viana Cruz

\section{Coordenadora do Evento}

Andréa Telino Gomes

\section{Organizadores}

Academics - Eventos acadêmicos online

Editora Omnis Scientia

Andréa Telino Gomes

Daniel Luís Viana Cruz

Alex Aguiar da Silva

\section{Palestrantes}

Alex Aguiar da Silva

André Búrigo Leite

Cecília Aguiar da Silva

Eugênio Bastos da Costa

Jorge Recarte Henriquez Guerrero

Renan Ferreira da Rocha

\section{Avaliadores}

Alex Aguiar da Silva

Helio Cardoso Martim

Péricles Felipe Bastos Gomes

\section{Imagem de Capa}

Freepik

\section{Revisão}

Os autores

\section{(9) $\mathbb{\oplus \Theta \Theta}$}

Este trabalho está licenciado com uma Licença Creative Commons - Atribuição-NãoComercialSemDerivações 4.0 Internacional.

O conteúdo abordado nos artigos, seus dados em sua forma, correção e confiabilidade são de responsabilidade exclusiva dos autores. 


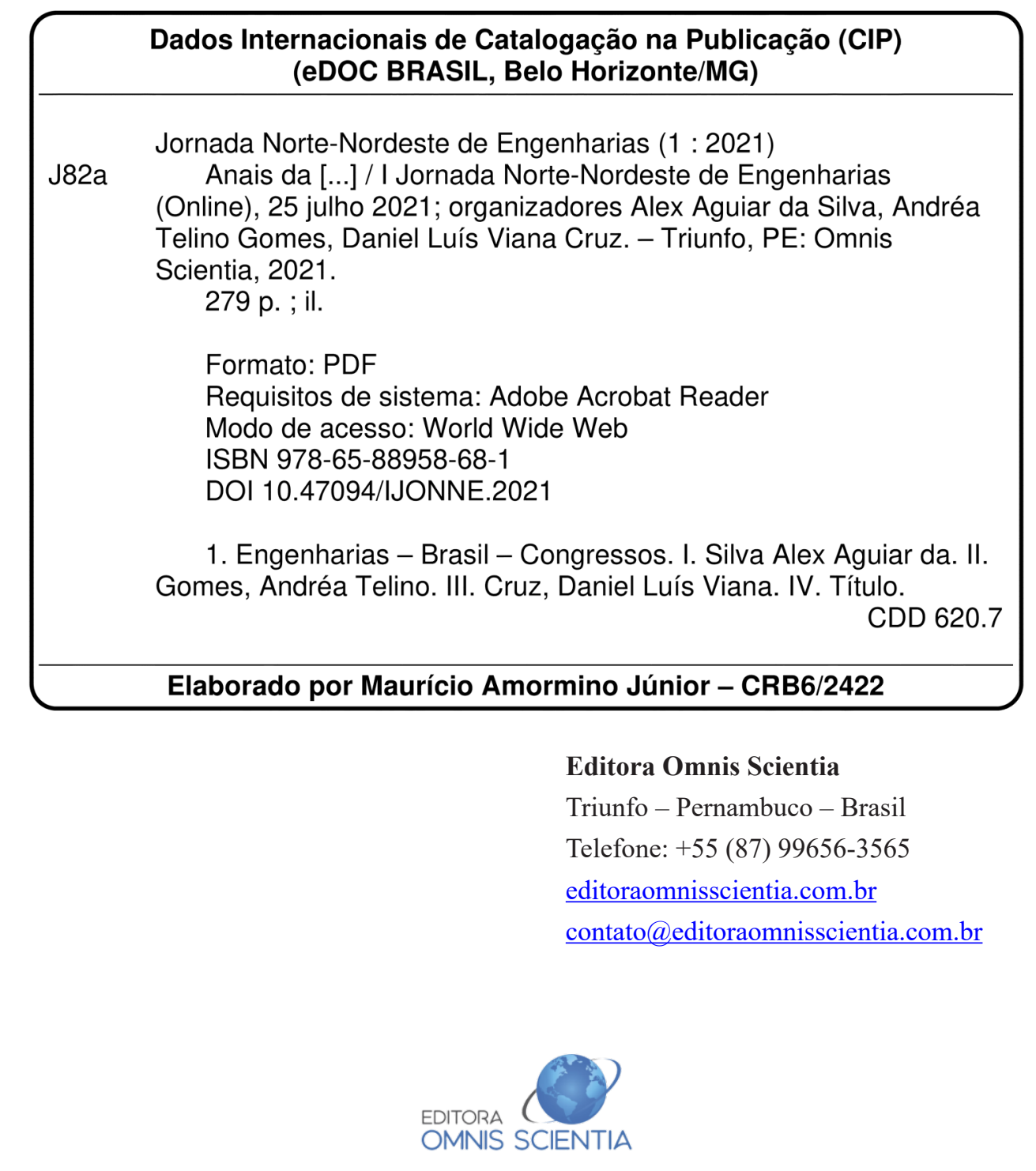




\section{EDITORIAL}

A grande área das engenharias abrangente uma série de ramos específicos, cada qual com determinados campos de aplicação e tipos de tecnologias, conjugando conhecimentos especializados no sentido de viabilizar as utilidades, tendo em conta a sociedade, a técnica, a economia e o meio ambiente. Deste modo, as engenharias aplicam o conhecimento científico, econômico, social e prático, para criar, desenhar, construir, manter e melhorar estruturas, máquinas, aparelhos, sistemas, materiais e processos. Assim, se adquire e se aplicam os conhecimentos matemáticos e técnicos na invenção, aperfeiçoamento e implementação de utilidades que realizem uma função ou objetivo.

A I Jornada Norte-Nordeste de Engenharias (online) I JONNE, objetivou reunir e integrar as engenharias para promover e apoiar esta área do conhecimento por meio de palestras, submissões de trabalhos na modalidade de resumos expandidos e exposição dos resumos aprovados.

Os participantes receberam certificados de participação de 20 horas e foi concedido menção honrosa para os três melhores trabalhos. Seguem os títulos dos resumos que foram premiados:

Modalidade resumo expandido

387210 - DIMENSIONAMENTO DE TROCADOR DE CALOR A PLACAS PARA PASTEURIZAÇÃO DE LEITE PELO PROCESSO HTST

387222 - DESENVOLVIMENTO DE UM DISPENSER DE ÁLCOOL GEL AUTOMÁTICO PARA MONITORAMENTO DA COMPLACÊNCIA DE MÃOS

387225 - REDE NEURAL ARTIFICIAL PARA VISCOSIDADE E CONDUTIVIDADE TÉRMICA DO FLUIDO REFRIGERANTE R32

A equipe organizadora da I JONNE agradece a todos os participantes, palestrantes e avaliadores pela participação e colaboração no congresso. 


\section{SUMÁRIO}

\section{ENGENHARIA CIVIL}

ANÁLISE DO USO DE RESÍDUO DE BRITAGEM DAS ROCHAS EM CONCRETO AUTOADENSÁVEL: UMA REVISÃO DA LITERATURA.....................................................12

ESTRATÉGIAS PARA A PREVENÇÃO DE ACIDENTES NA CONSTRUÇÃO CIVIL: UMA REVISÃO SISTEMÁTICA DA LITERATURA.

RESISTÊNCIA À COMPRESSÃO DE MISTURAS DE SOLO-CIMENTO COM NANOSSÍLICA E SÍLICA ATIVA.

PATOLOGIAS EM OBRAS PARALISADAS: O CASO DO ESTÁDIO COLOSSO DO TAPAJÓS NA CIDADE DE SANTARÉM - PA.

DIFERENÇA NO CUSTO DE MÃO DE OBRA EM FABRICAÇÃO DE LAJES PRÉ- MOLDADAS EM AMBITO NACIONAL

ESTUDO COMPARATIVO DE FUNDAÇÕES PARA UM EDIFÍCIO MODELO EM JOÃO PESSOA/PB: SAPATA X ESTACA HÉLICE CONTÍNUA.

MANIFESTAÇÕES PATOLÓGICAS EM EDIFICAÇÕES COM PAREDES DE CONCRETO ARMADO: ESTUDO DE CASO EM OBRA NA CIDADE DE JOÃO PESSOA - PB.....

REUTILIZAÇÃO DO PÓ RESIDUAL DE MARMORARIA PARA SUBSTITUIÇÃO DO AGREGADO MIÚDO NO CONCRETO.

SUBSIDÊNCIA OCORRIDA NOS BAIRROS PINHEIRO, BEBEDOURO E MUTANGE EM MACEIÓ - AL: UMA REVISÃO BIBLIOGRÁFICA.

CARACTERIZAÇÃO FÍSICA DAS VARIAÇÕES DIMENSIONAIS DO BAMBU GUADUA WEBERBAUERI DE RIO BRANCO - AC.

ESTUDO DA OCORRÊNCIA DE SOLOS COLAPSÍVEIS NA REGIÃO NORDESTE DO BRASIL.

OS EFEITOS DO USO DE POÇOS ARTESIANOS NO PROCESSO DE SALINIZAÇÃO COSTEIRA NA CIDADE DE SÃO LUÍS: UM LEVANTAMENTO BIBLIOGRAFICO.

TEORIADOS SÓLIDOS CELULARES EMPREGADOS EM REFORÇOS PARAACONSTRUÇÃO CIVIL

AVALIAÇÃO DO EXTRATO DE BOLDO E AROEIRA COMO INIBIDOR VEGETAL DE BAIXO CUSTO......

ANÁLISE DE DOSAGEM DE MATRIZ CIMENTÍCIA COM FIBRAS DE POLIPROPILENO: REVISÃO DA LITERATURA 
ANÁLISE DA RETENÇÃO DE CLORETOS EM ARGAMASSAS COM RESÍDUOS DE CELULOSE.

\section{ENGENHARIA DE MATERIAIS E METALÚRGICA}

ANÁLISE DA DEGRADAÇÃO FERRÍTICA DO AÇO INOXIDÁVEL DUPLEX SAF 2205 SUBMETIDO A ENVELHECIMENTO ISOTÉRMICO.

ANÁLISE DE INCLUSÕES E QUANTIFICAÇÃO DE POROSIDADE DO BRONZE AO ESTANHO EM ESTRUTURA BRUTA DE FUSÃO. 100

SÍNTESE RÁPIDA DE NANOFIOS DE NIOBATO DE SÓDIO. 105

AVALIAÇÃODAINCORPORAÇÃODACAREPADELAMINAÇÃOCOMOPARTEDAMATÉRIAPRIMA NA PRODUÇÃO DE SÍNTER DE FINOS DO MINÉRIO DE FERRO. 110

ESTUDO PROSPECTIVO DO PETRÓLEO APLICADO EM NANOMATERIAL CERÂMICO...... 115

ESTUDO PROSPECTIVO DE NANOMATERIAL CERÂMICO APLICADO EM SAÚDE MÉDICA E DENTÁRIA.

ESTUDO PROSPECTIVO DE MAGNETITA NANOMÉTRICA COMO MATERIAL FERROMAGNETICO.

ESTUDO PROSPECTIVO DE CERÂMICA AVANÇADA UTILIZANDO DOPAGEM......

BUSCA DE ANTERIORIDADE DE CERÂMICA APLICADA NA REMOÇÃO DE CONTAMINANTE..... .131

APLICAÇÕES DE $\mathrm{MoO}_{3}$ OBTIVO ATRAVÉS DE PRECIPITAÇÃO. 136

REVISÃO BIBLIOGRÁFICA SOBRE TUNGSTATO DE BÁRIO EM MEIO AQUOSO. 140

ESTUDOS DA DIFUSÃO DE CARBONO NOS AÇOS SAE 1020 E 1045 SUBMETIDOS À TRATAMENTO TERMOQUÍMICO DE CEMENTAÇÃO SÓLIDA. 145

\section{ENGENHARIA DO PRODUTO}

DESENVOLVIMENTO DE UM DISPENSER DE ÁLCOOL GEL AUTOMÁTICO PARA MONITORAMENTO DA COMPLACÊNCIA DE MÃOS. 150

\section{ENGENHARIA ELÉTRICA}

ENERGIA EÓLICA NO CEARÁ .156

\section{ENGENHARIA HIDRÁULICA}

ESTUDO DE MÉTODOS ANALÍTICOS PARA DETERMINAÇÃO DE PERDA DE CARGA EM TUBULAÇÕES DE PVC UTILIZADAS EM IRRIGAÇÕES. 162 
DESENVOLVIMENTO DE PRÁTICAS LABORATORIAIS APLICANDO OS CONCEITOS DE FENÔMENOS DE TRANSPORTES. 168

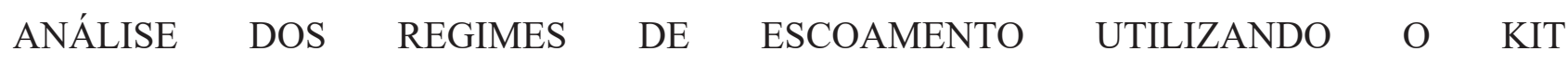
AQUALIBRIUM...

\section{ENGENHARIA MECÂNICA}

AVANÇO NO EMPREGO DE GABARITOS DE SOLDAGEM NO PROCESSO DE FABRICAÇÃO DE CHASSIS FORMULA SAE..

ANÁLISE DA DISTRIBUIÇÃO DE TEMPERATURA NOS PNEUS DE UM VEÍCULO FORMULA SAE PARA DIFERENTES CARGAS VERTICAIS. 184

\section{ENGENHARIA QUÍMICA}

UTILIZAÇÃO DE OXIDAÇÃO AVANÇADA PARA O TRATAMENTO DE EFLUENTES INDUSTRIAIS: UMA REVISÃO DA LITERATURA..

ANÁLISE DOS PRINCIPAIS PROCESSOS DE PRODUÇÃO DE BIODIESEL: UMA REVISÃO DE LITERATURA.

A QUÍMICA DOS AGROTÓXICOS USADOS EM AGRICULTURA: UMA REVISÃO DA LITERATURA. 201

AVALIAÇÃO CINÉTICA DA REAÇÃO DE DEGRADAÇÃO DE DIURON PRESENTE EM ÁGUAS SUPERFICIAIS.

ESTUDO DE CASO DO TRATAMENTO DE GÁS ÁCIDO COM COLUNA DE ABSORÇÃO DE LEITO RECHEADO.

ELABORAÇÃO DA CURVA DE CALIBRAÇÃO PARA ACRILAMIDA PELO MÉTODO DE CROMATOGRAFIA LÍQUIDA DE ALTA EFICIÊNCIA.

DETERMINAÇÃODECONSTANTESCINÉTICASPELOS MÉTODOSINTEGRALEDERUNGEKUTTA PARA REAÇÃO DE OXIDAÇÃO DO ANTRACENO.

DIMENSIONAMENTO DE TROCADOR DE CALOR A PLACAS PARA PASTEURIZAÇÃO DE LEITE PELO PROCESSO HTST.. 229

REDE NEURAL ARTIFICIAL PARA VISCOSIDADE E CONDUTIVIDADE TÉRMICA DO FLUIDO REFRIGERANTE R32.

TRANSFORMAÇÃO DO POLITEREFTALATO DE ETILENO RECICLADO EM FIBRAS TÊXTEIS DE POLIÉSTER. .239

\section{ENGENHARIA SANITÁRIA}

O USO DA ENERGIA SOLAR EM HOSPITAIS EM TEMPOS DE PANDEMIA 
ESTUDO DE VARIÁVEIS QUE POSSAM INFLUENCIAR NO CONSUMO DE ÁGUA DA CIDADE DE BAIXO GUANDÚ-ES........

\section{OUTRAS}

SISTEMAAUTOMÁTICO DE IRRIGAÇÃO. .260

A FRUTA DO MILAGRE - SYNSEPALUM DULCIFICUM.. 266 FOSSAS DE EVAPOTRANSPIRAÇÃO: UMA SOLUÇÃO SUSTENTÁVEL PARA OS DEFICITS NO SANEAMENTO EM ÁREAS CARENTES. .271

PROJETO DE ROBÓTICA SOCIAL E EDUCACIONAL .276 


\title{
CARACTERIZAÇÃO FÍSICA DAS VARIAÇÕES DIMENSIONAIS DO BAMBU GUADUA \\ WEBERBAUERI DE RIO BRANCO - AC
}

\author{
Tiago Henrique da Costa Viana1; Gabriela Grotti Silveira², Pedro Bomfim Segobia ${ }^{3}$, José \\ Roberto de Lima Murad ${ }^{4}$ \\ ${ }_{23}$ Tiago Henrique da Costa Viana, UFAC, Rio Branco, Acre. \\ 24 Gabriela Grotti Silveira, Faculdade Unyleya, Rio de Janeiro, Rio de Janeiro. \\ ${ }^{3}$ Pedro Bomfim Segobia, UnB, Brasília, Distrito Federal. \\ ${ }^{4}$ José Roberto de Lima Murad, UFAC, Rio Branco, Acre
}

DOI: 10.47094/IJONNE.2021.3

\begin{abstract}
RESUMO
Com a demanda crescente de materiais de construção que atendam aos parâmetros característicos do desenvolvimento sustentável há a necessidade do estudo de materiais alternativos, como o bambu. Objetivando conhecer as características das variações dimensionais, bem como as interferências das regiões do colmo do bambu Guadua weberbaueri, desenvolve-se o presente estudo. Tal objetivo foi alcançado através de execução de corpos de prova e de ensaios laboratoriais com imersão em água e medições para obtenção das variações. Os dados foram, então, obtidos e analisados com relação a outros estudos. Os dados significativos expressaram características relativas ao comportamento dimensional do bambu, em imersão de água, o que revela, a longo prazo, aspectos importantes que podem servir como base para caracterizações do bambu para possível uso construtivo.
\end{abstract}

PALAVRAS-CHAVE: Bambu. Variação Dimensional. Guadua weberbaueri. ÁREA TEMÁTICA: Engenharia Civil.

\section{INTRODUÇÃO}

As madeiras constituem uma das primeiras formas dominadas pelo ser humano para suas construções primitivas, segundo Padovan (2010). Na construção civil, de acordo com Júnior, Kenupp e Campos (2009), a extração de madeira é expressiva e, aliada a todos os outros processos do setor construtivo, é responsável por uma alta emissão de carbono, de ordem mundial. Apesar deste cenário desfavorável, permanece ainda a tendência à utilização de materiais convencionais (concreto, aço e cimento), conforme Tatibana, Reis e Bianchi (2016). Paralela e contrária à situação vivenciada pelo Brasil, os países asiáticos apresentam um histórico consolidado no uso de bambu 
em sua forma natural, desde suas construções vernaculares (PADOVAN, 2010).

À exemplo de tais países, e para que o Brasil seja capaz de usufruir da utilização do bambu a nível industrial na construção civil, são necessários estudos de cunho técnico e científico (GHAVAMI e MARINHO, 2005). Desta forma, o presente estudo visa avaliar o desempenho físico, por meio de ensaios não destrutivos de imersão e secagem em estufa, do bambu Guadua weberbaueri, visando a obtenção de informações sobre o comportamento da variação dimensional para inchamento e retração, possibilitando a utilização ou não, desse material e sua aplicação na engenharia civil como material sustentável e economicamente viável.

\section{METODOLOGIA}

Para a execução dos ensaios, foram utilizados: paquímetro analógico para aferição das variações; corpos de prova para execução do ensaio; recipientes para imersão dos corpos de prova em água e estufa.

A sequência das etapas de realização do ensaio de determinação das variações dimensionais necessitou etapas de confecção e molde de corpos de prova e, em laboratório, ocorreu conforme expresso:

1. Foram fixados pares de pontos com caneta esferográfica em faces opostas ao longo da direção longitudinal, circunferencial e radial;

2. As dimensões dos corpos de prova secos ao ar foram medidas com um paquímetro e posteriormente os mesmos foram imersos na água;

3. A cada vinte e quatro horas são feitas novas medições, durante o período de sete dias;

4. As variações em relação às dimensões secas (V) são obtidas pela seguinte equação:

$$
V=\left(\frac{D_{v}-D_{s}}{D_{s}}\right) * 100
$$

5. Após pesagens dos corpos saturados, foram levados a estufa, onde secos, foram medidas suas dimensões. 


\section{RESULTADOS E DISCUSSÕES}

Com base na execução das etapas supracitadas, foi possível, a partir dos resultados médios calculados e das leituras realizadas ao longo dos sete dias, construir a variação dimensional média, conforme região do bambu e sentido dimensional (Tabela 1).

Tabela 1: Valores de variação média para cada sentido, para inchamento, em função da região do bambu.

\begin{tabular}{cccc}
\hline Região do Colmo & Longitudinal (\%) & Circunferencial (\%) & Radial (\%) \\
\hline Base & 0,64 & 2,79 & 7,84 \\
\hline Meio & 0,60 & 3,27 & 9,53 \\
\hline Topo & 0,80 & 1,96 & 13,55 \\
\hline
\end{tabular}

Fonte: autor, 2019.

Da Tabela 1, construiu-se então o gráfico de variações dimensionais em função da região do bambu, sintetizadas na Figura 1:

Figura 1: Distribuição de variações dimensionais, conforme região do bambu, em cada sentido.

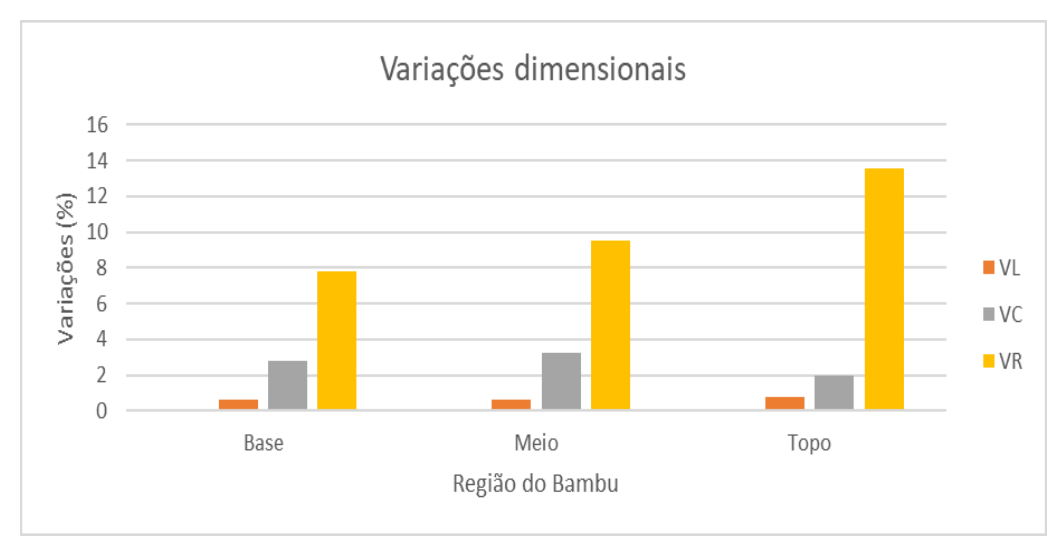

Fonte: autor, 2019.

A partir do gráfico acima, é possível notar que as Variações Radiais (VR) foram superiores nas três regiões do colmo, sendo preponderantes, na região do topo. A Variação Longitudinal (VL) foi relativamente de baixa significância pela expressão dos menores valores em contrapartida à Variação Circunferencial (VC), que teve maior valor na região do meio do colmo. 
Para a variação das dimensões quando o corpo de prova foi submetido à secagem em estufa, originaram-se os dados na Tabela 2 e Figura 2.

Tabela 2: Valores de variação média para cada sentido, em função da região do bambu, por retração.

\begin{tabular}{cccc}
\hline Regiões do Colmo & $\mathbf{V I}(\mathbf{\%})$ & $\mathbf{V c}(\mathbf{\%})$ & $\mathbf{V r}(\mathbf{\%})$ \\
\hline Base & 0,18 & $-3,96$ & $-1,73$ \\
\hline Meio & 0,08 & -3 & $-3,56$ \\
\hline Topo & 0,36 & $-1,27$ & $-6,09$ \\
\hline
\end{tabular}

Fonte: autor, 2019.

Figura 2: Distribuição de retração dimensional conforme região do colmo e sentido dimensional

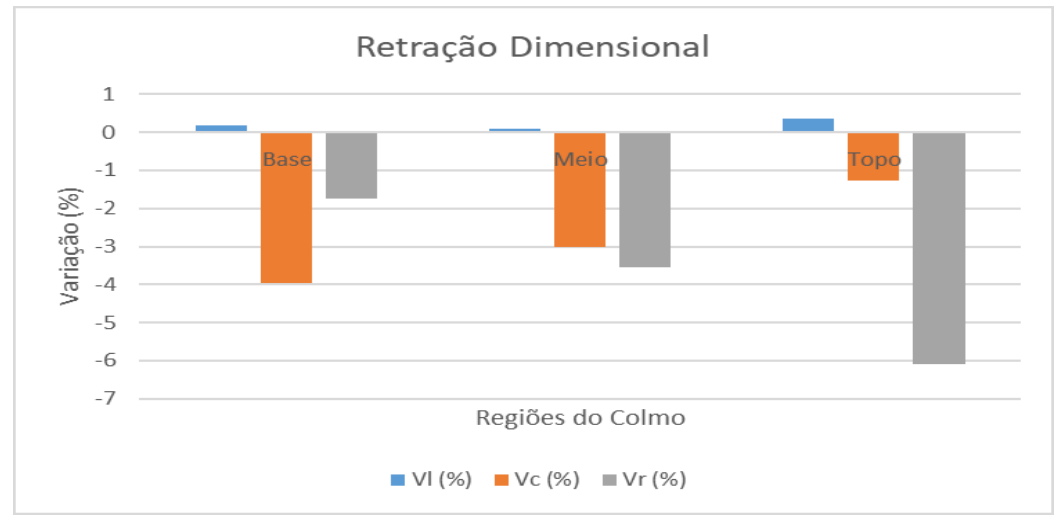

Fonte: autor, 2019.

Para a situação expressa na Figura 2, é possível notar discrepâncias nos resultados. Nas regiões de topo e base, as variações foram divergentes, sendo $\mathrm{VC}$ superior às demais, para a região basal, em contrapartida à elevada VR expressa na região de topo. Na região do meio, VC e VR tem expressões semelhantes. Para as três regiões, no entanto, VL teve crescimento em sentido oposto, caracterizando inchamento. Esta variação em sentido contrário poder-se-á relacionar-se com o fato de ter sido realizada secagem em estufa após o ensaio de absorção de água, havendo alteração nos resultados de retração dos corpos de prova.

Dos resultados expressos anteriormente, pode-se notar que, conforme estabelece Murad (2011), ao realizar similar ensaio de variações dimensionais para o mesmo tipo de bambu, podemos obter resultados semelhantes, com leves variações, para os dados de variações dimensionais por inchamento.

Para a retração, no entanto, o comportamento não foi o mesmo que o obtido por Murad (2011), devido o sentido longitudinal ter tido variação contrária ao esperado, pois no estudo do autor, todos os sentidos sofrem retração. Para os sentidos circunferencial e radial, os valores seguiram o comportamento de retração esperado. Como citado anteriormente, essa variação 
longitudinal em sentido contrário, pode ser explicada pelo fato de ter sido utilizado os mesmos corpos de prova do ensaio de absorção de água, que foram levados a estufa após a saturação e não em condição de secos ao ar, fato que pode ter modificado a retração longitudinal esperada.

\section{CONSIDERAÇÕES FINAIS OU CONCLUSÃO}

Com a análise realizada, foi possível notar a constância de valores obtidos, o que serve como parâmetro para caracterização física dimensional e principalmente, a longo prazo, como base para demais caracterizações científicas do bambu Guadua Weberbaueri, a ponto de, com seu estudo, torná-lo futuramente um material prático e eficiente para utilização na construção civil.

\section{REFERÊNCIAS BIBLIOGRÁFICAS}

GHAVAMI, K.; MARINHO, A. B. Propriedades físicas e mecânicas do colmo inteiro do bambu da espécie Guadua angustifolia. Revista Brasileira de Engenharia Agrícola e Ambiental, v.9, n.1, p.107-114, 2005.

PADOVAN, R. B. O Bambu na Arquitetura: Design de Conexões Estruturais. Universidade Estadual Paulista Júlio de Mesquita Filho. Bauru, São Paulo - SP. 2010.

TATIBANA, R. M; REIS, M. P dos; BIANCHI, G. Bambu como Matéria-Prima para Construções Sustentáveis. Periódico TécnicoCientífico Cidades Verdes, v. 04, pp. 95-103, nº 10, 2016.

MURAD, J. R. L. Estudo Experimental das Propriedades Físicas, Mecânicas e Aplicações Estruturais do Bambu Guadua Spp de Assis Brasil - Ac. Tese. Universidade Federal Fluminense UFF. Rio de Janeiro - RJ, 2011. 


\section{editoraomnisscientia@gmail.com M} https://editoraomnisscientia.com.br/

@editora_omnis_scientia @ https://www.facebook.com/omnis.scientia.9 f

$$
\text { +55 (87) 9656-3565 @ }
$$




\section{editoraomnisscientia@gmail.com M} https://editoraomnisscientia.com.br/ $\oplus$

@editora_omnis_scientia@ https://www.facebook.com/omnis.scientia.9 f

$$
\text { +55 (87) 9656-3565 @ }
$$

